\title{
Negative and positive control ranges in the bacterial reverse mutation test: JEMS/BMS collaborative study
}

\author{
Masayuki Kato ${ }^{1 *} \mathbb{D}$, Kei-ichi Sugiyama ${ }^{2}$, Toshiro Fukushima ${ }^{3}$, Yasuyoshi Miura ${ }^{3}$, Takumi Awogi ${ }^{4}$, Shigetomo Hikosaka ${ }^{5}$, \\ Kumiko Kawakami ${ }^{6}$, Madoka Nakajima ${ }^{7}$, Masato Nakamura ${ }^{8}$, Hajime Sui $^{6}$, Kumiko Watanabe $^{9}$ and Atsushi Hakura ${ }^{10}$
}

\begin{abstract}
A large-scale study was conducted by multiple laboratories affiliated with the Japanese Environmental Mutagen Society and the Bacterial Mutagenicity Study Group to investigate possible proficiency indicators for the bacterial reverse mutation test with a preincubation procedure. Approximately 30 laboratories generated negative and positive control count data and dose-response curves of the positive control articles for the bacterial reverse mutation test, with assays conducted annually from 2013 to 2016. Overall, the majority of the negative and positive control counts for Salmonella Typhimurium strains TA100, TA1535, TA98, and TA1537, and Escherichia coli strain WP2UvrA, with and without S9 mix, were within the range of the means $\pm 2 \times$ standard deviation. The negative counts were normally distributed (strains TA100, TA98, and WP2uvrA) or followed Poisson distribution (strains TA1535 and TA1537), and the positive control counts for all strains were approximately normally distributed. In addition, the distribution of the negative and positive control counts was relatively constant over the 4 years. The number of revertant colonies increased in a dose-dependent linear or exponential fashion up to the recommended doses for the respective positive control articles in Japan. These data are valuable for determining the acceptance criteria and an estimation of the laboratory proficiency for the bacterial reverse mutation test.
\end{abstract}

Keywords: Bacterial reverse mutation test, Validation study, Negative control range, Positive control range

\section{Background}

The bacterial reverse mutation test, known as the Ames test, is often used to identify and characterize the mutagenicity of chemicals in basic research, and to examine the safety of industrial products prior to approval by regulatory agencies [1-6]. The structural alerts for mutagenicity derived from the results are also used by regulatory agencies to predict mutagenic impurities using in silico analysis according to the International Council for Harmonisation of Technical Requirements for Pharmaceuticals for Human Use M7 guideline [7]. The bacterial reverse mutation test is an integral component of genotoxicity tests performed as part of the regulatory

\footnotetext{
* Correspondence: masayuki-kato@cmic.co.jp

${ }^{1}$ CMIC Bioresearch Center, CMIC Pharma Science Co., Ltd., Hokuto-shi,

Yamanashi 408-0044, Japan

Full list of author information is available at the end of the article
}

requirements in accordance with the principles of Good Laboratory Practice, and therefore, it is important to use appropriate indicators for evaluating and demonstrating laboratory proficiency in the test. For this purpose, the negative and positive control ranges, along with the doseresponse relationships of the positive control articles, are considered to be promising candidates.

To refine and maintain the data quality and experimental techniques used for the reverse mutation test, the Japanese Environmental Mutagen Society (JEMS)/ Bacterial Mutagenicity Study Group (BMS) collaboratively conducted validation studies over two 4-year periods (2006-2009 and 2013-2016). In this paper, we report the results obtained from the four JEMS/BMS validation studies conducted annually during the period 2013-2016. The number of participating laboratories per year was 36 in 2013, 30 in 2014, 27 in 2015, and 26 


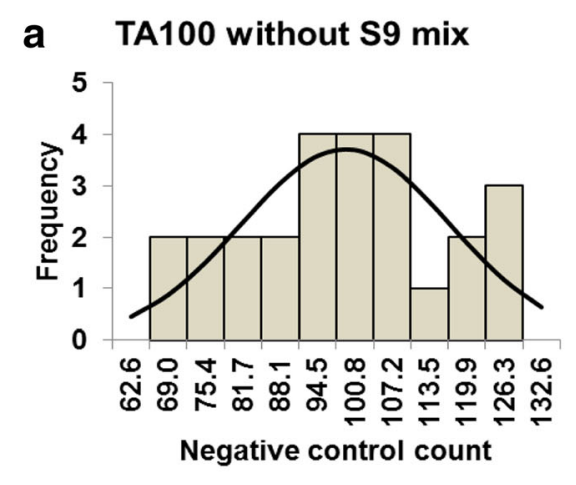

C
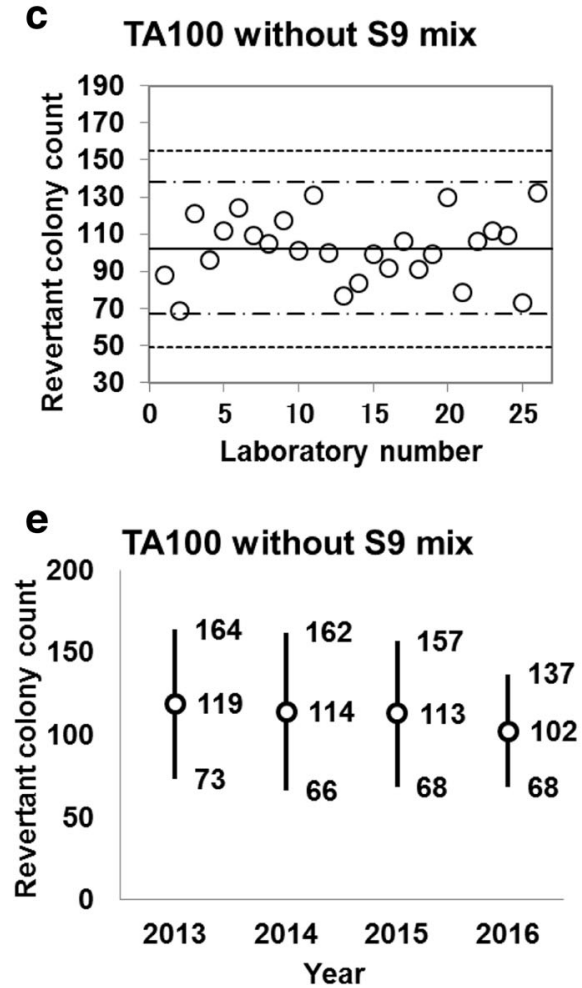

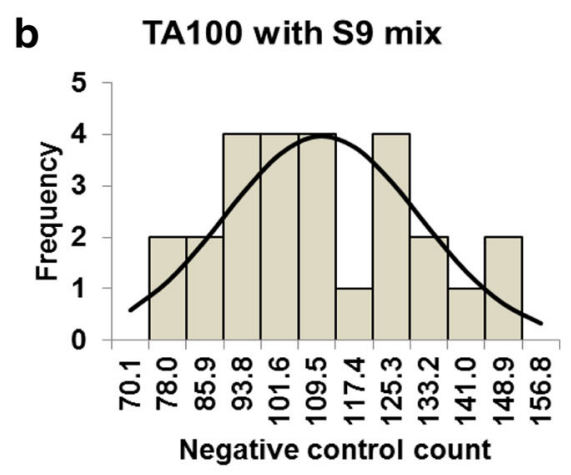

d

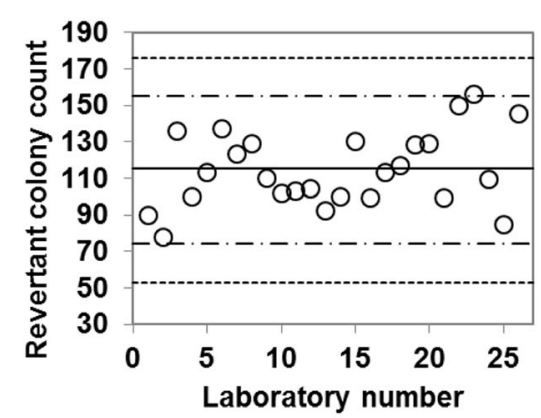

$\mathbf{f}$

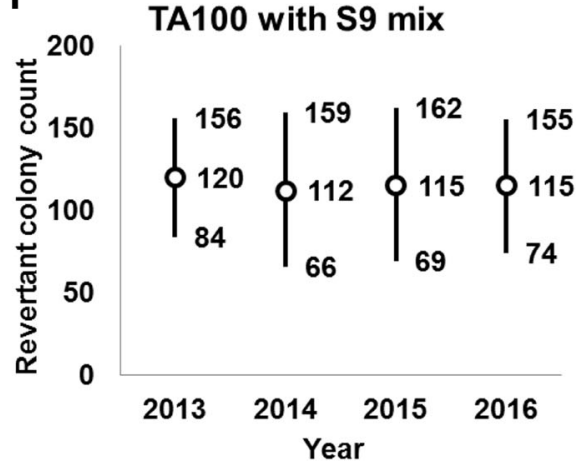

Fig. 1 Negative control counts, and their subsequent analysis, for Salmonella Typhimurium strain TA100 with and without S9 mix. Histograms show the negative control counts, and the curves indicate the expected values calculated based on the assumption that the negative control counts follow a normal distribution, without (a) and with (b) 59 mix. Scatter plots showing the negative control counts generated by each participating laboratory without (c) and with (d) S9 mix are also shown, where the inner horizontal lines (---) indicate the mean $\pm 2 \times$ standard deviation (SD), and outer horizontal lines (--) indicate the mean $\pm 3 \times$ SD. The data shown in panels (a) to (d) are taken from the study conducted in 2016 . The mean $\pm 2 \times$ SD values for the negative control counts for each individual year without (e) and with (f) S9 mix are also presented

in 2016. Data were obtained for the negative and positive control counts and the dose-response curves of the respective positive control articles using the five tester strains with and without $\mathrm{S} 9$ mix. The positive control articles (AF-2, 2-(2-furyl)-3-(5-nitro-2-furyl) acrylamide; SA, sodium azide; 9AA, 9-aminoacridine hydrochloride; and 2AA, 2-aminoanthracene) and bacterial strains (Salmonella enterica subsp. enterica serovar Typhimurium strains TA100, TA1535, TA98, and TA1537, and Escherichia coli strain WP2uvrA) used were those recommended in the Organisation for Economic Co-operation and Development (OECD) guideline for the testing of chemicals 471 [6].

\section{Materials and methods}

Chemicals and materials

An Ames Test Positive Control AM Multi-set (Lot number M0048, Wako Pure Chemical Industries, Ltd., Osaka, Japan) was used to provide the positive controls. The set comprised 2-(2-furyl)-3-(5-nitro-2-furyl) 

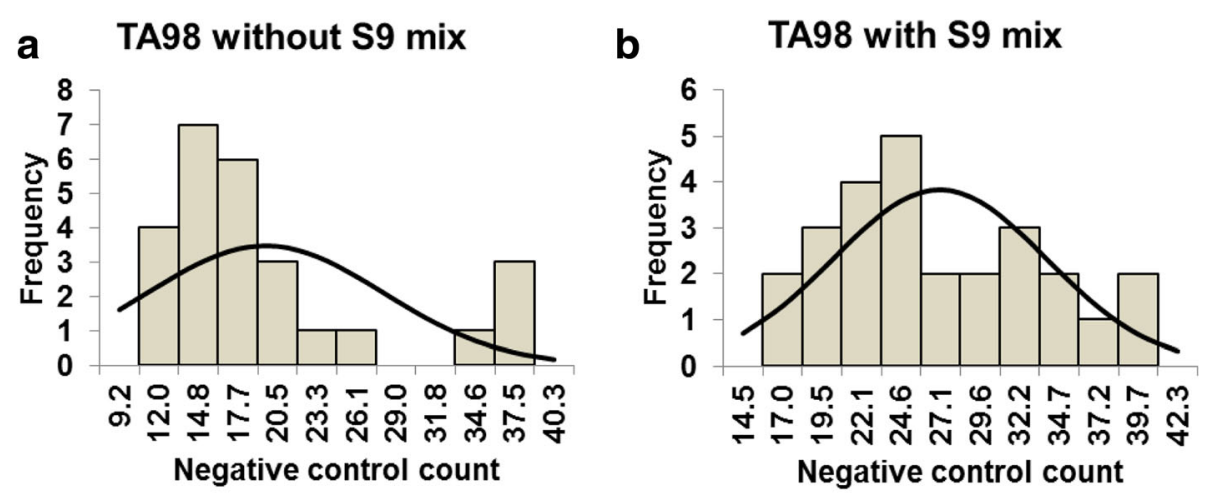

C TA98 without $\mathbf{S 9}$ mix

d

TA98 with $\$ 9$ mix
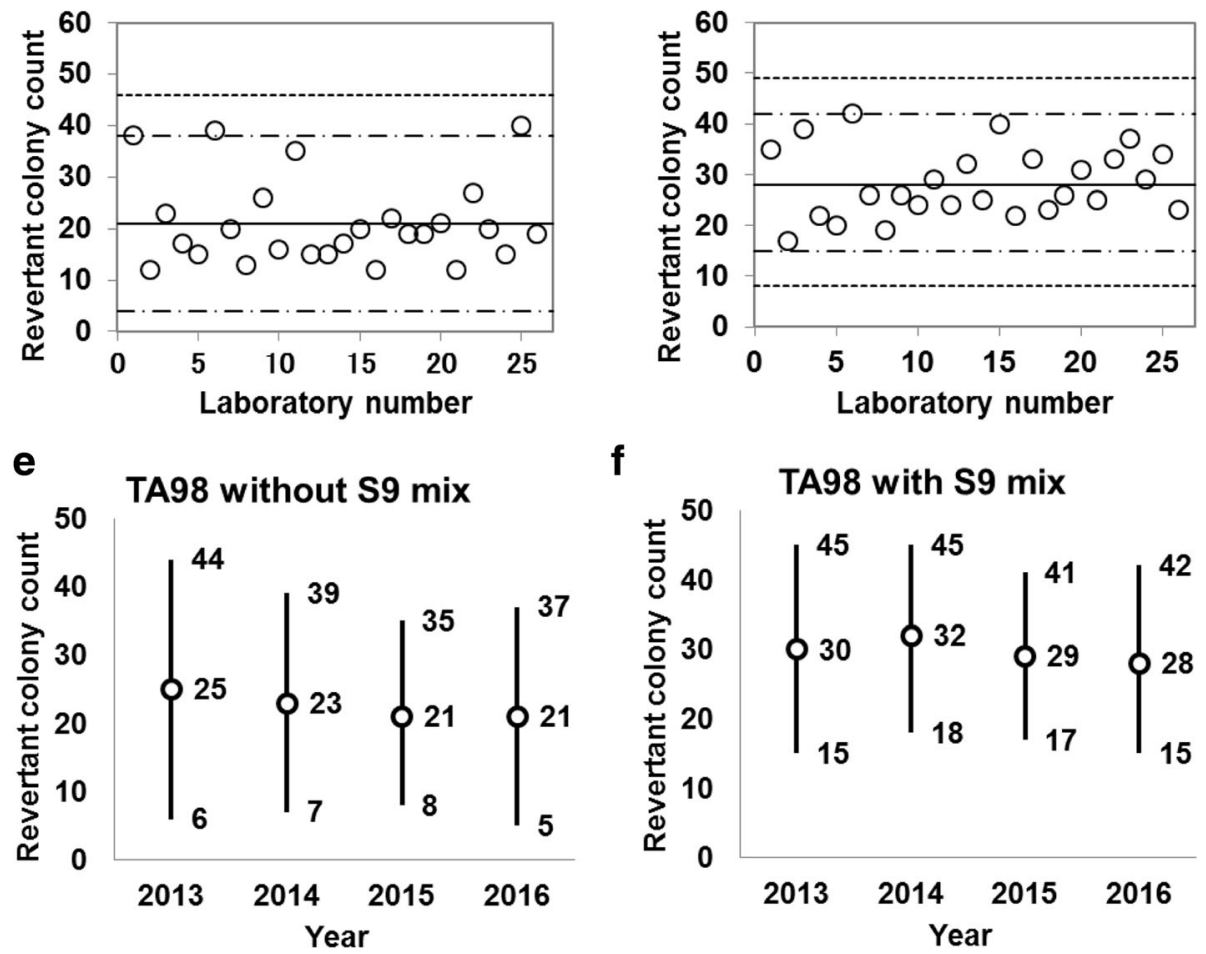

f

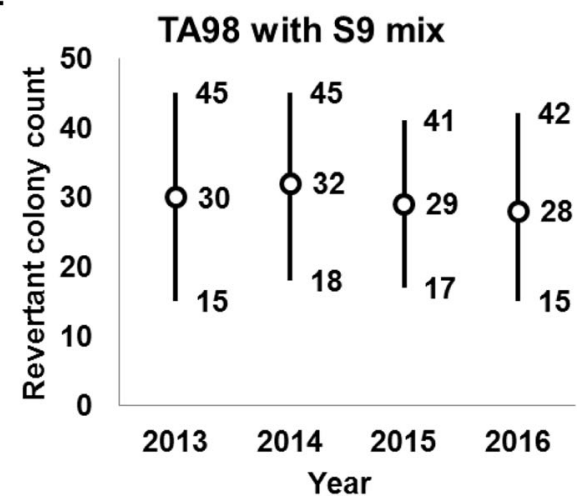

Fig. 2 Negative control counts, and their subsequent analysis, for Salmonella Typhimurium strain TA98 with and without S9 mix. Scatter plots showing the negative control counts generated by each participating laboratory without (c) and with (d) S9 mix are also shown, where the inner horizontal lines (---) indicate the mean $\pm 2 \times$ standard deviation (SD), and outer horizontal lines (--) indicate the mean $\pm 3 \times$ SD. The data shown in panels (a) to (d) are taken from the study conducted in 2016. The mean $\pm 2 \times$ SD values for the negative control counts for each individual year without (e) and with (f) S9 mix are also presented

acrylamide (AF-2, purity of $99.7 \%)$, sodium azide (SA, purity of $100.0 \%)$, 9-aminoacridine hydrochloride (9AA, purity of 99.4\%), and 2-aminoanthracene (2AA, purity of $96.7 \%)$. AF-2, 9AA, and $2 \mathrm{AA}$ were dissolved in dimethyl sulfoxide (DMSO, purity of 100\%; Wako Pure Chemical Industries), while SA was dissolved in purified water.

S9 fraction, prepared from phenobarbital/5,6-benzoflavone-pretreated male Sprague-Dawley rat liver, was purchased from Oriental Yeast Co., Ltd. (Tokyo, Japan) or Kikkoman Biochemifa Co. (Chiba, Japan). Different lots of
S9 fraction were used throughout the 4-year study. The S9 mix used in the assays consisted of $10 \%(v / v) S 9$ fraction ( $1.0 \mathrm{mg}$ protein/plate), $100 \mathrm{mM} \mathrm{Na} \mathrm{HPO}_{4} / \mathrm{NaH}_{2} \mathrm{PO}_{4}$, $8 \mathrm{mM} \mathrm{MgCl} 2,33 \mathrm{mM} \mathrm{KCl}, 4 \mathrm{mM}$ NADP, $4 \mathrm{mM}$ NAD, and $5 \mathrm{mM}$ glucose-6-phosphate.

The $S$. Typhimurium top agar consisted of Bacto agar $(0.6 \%(w / v)$ final concentration) and $\mathrm{NaCl}(0.5 \%$ $(\mathrm{w} / \mathrm{v})$ final concentration) dissolved in purified water supplemented with $0.05 \mathrm{mM}$ L-histidine and $0.05 \mathrm{mM}$ D-biotin. The E. coli top agar was the same as that used for $S$. Typhimurium, minus the D-biotin. Minimum 


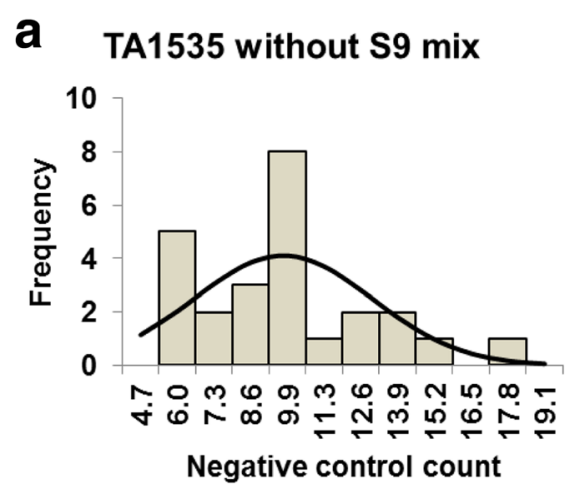

\section{TA1535 without S9 mix}
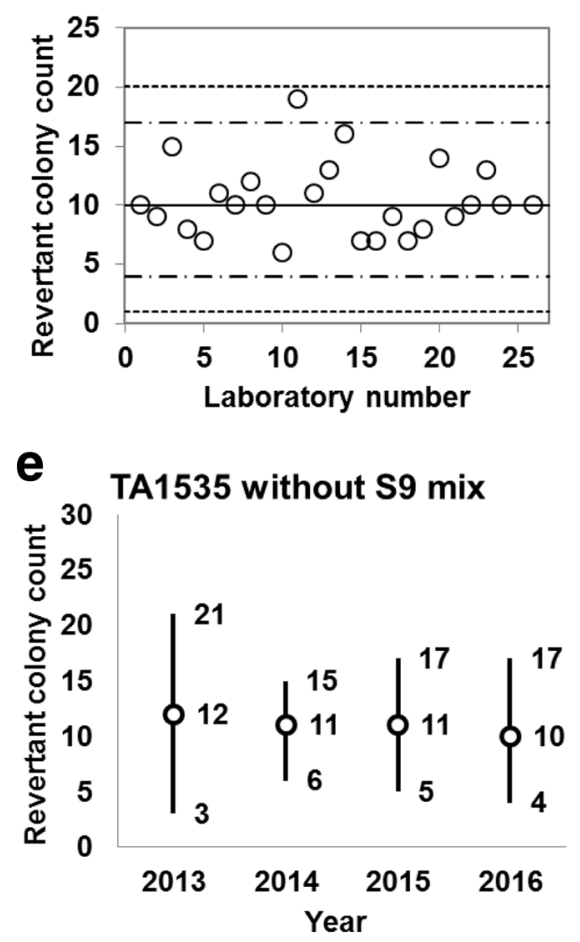

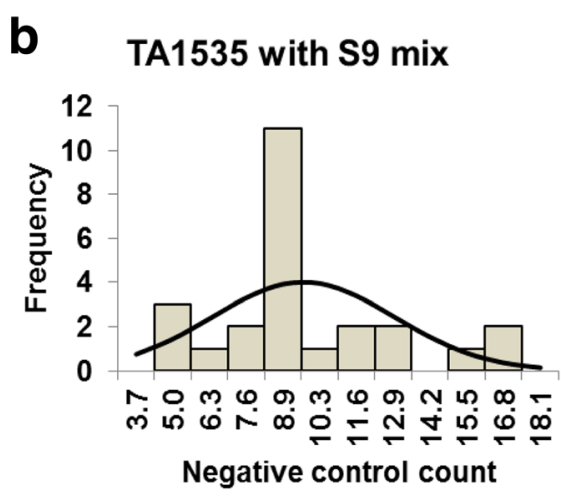

d

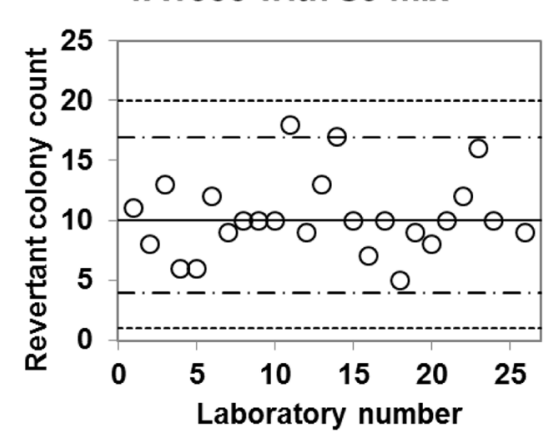

f

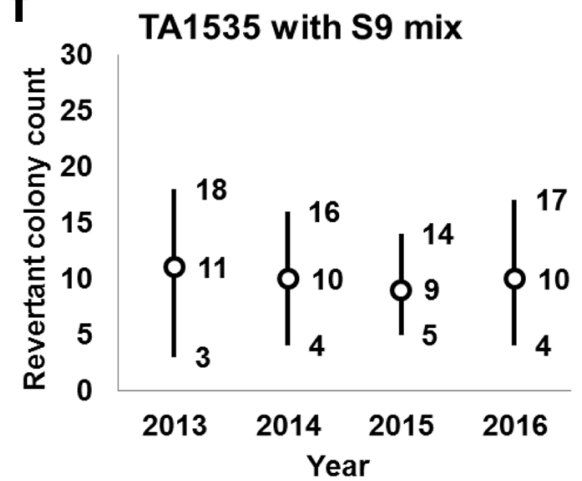

Fig. 3 Negative control counts, and their subsequent analysis, for Salmonella Typhimurium strain TA1535 with and without S9 mix. Scatter plots showing the negative control counts generated by each participating laboratory without (c) and with (d) S9 mix are also shown, where the inner horizontal lines (---) indicate the mean $\pm 2 \times$ standard deviation (SD), and outer horizontal lines (--) indicate the mean $\pm 3 \times$ SD. The data shown in panels (a) to (d) are taken from the study conducted in 2016. The mean $\pm 2 \times$ SD values for the negative control counts for each individual year without (e) and with (f) 59 mix are also presented

glucose agar plates were obtained from Oriental Yeast Co. or Kyokuto Pharmaceutical Industrial Co. (Tokyo, Japan).

\section{Bacterial strains}

The tester strains used were $S$. Typhimurium TA98

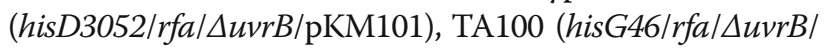
pKM101), TA1535 (hisG46/rfa/ $\Delta u v r B$ ), and TA1537 (hisC3076/rfa/ $u$ uvrB), and E. coli WP2uvrA (trpE/uvrA). These strains are recommended for use in the Ames test by Organisation for Economic Cooperation and Development (OECD) Guideline 471 [6], and this combination of strains is used in the majority of Japanese laboratories. Phenotypic characteristics of each of the strains, such as amino acid deficiencies (his for the Salmonella strains and trp for the E. coli strain), sensitivity to crystal violet ( $r f a)$, ampicillin resistance (pKM101), and sensitivity to ultraviolet light ( $\Delta u v r B$ for the Salmonella strains and $\triangle u v r A$ for the E. coli strain), were confirmed as described previously $[4,5,8,9]$ prior to use in each laboratory. In addition, the frozen stock culture of each strain was also confirmed to have responses within each laboratory's historical ranges to the negative and positive controls in advance of testing. 

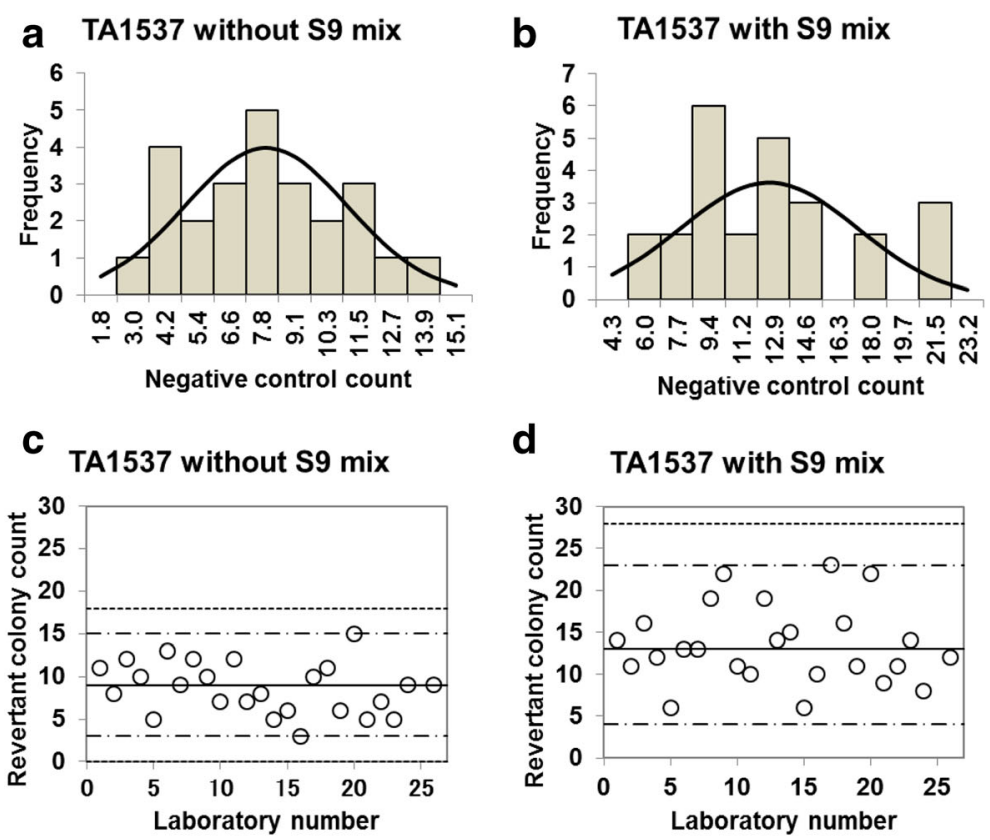

TA1537 with S9 mix
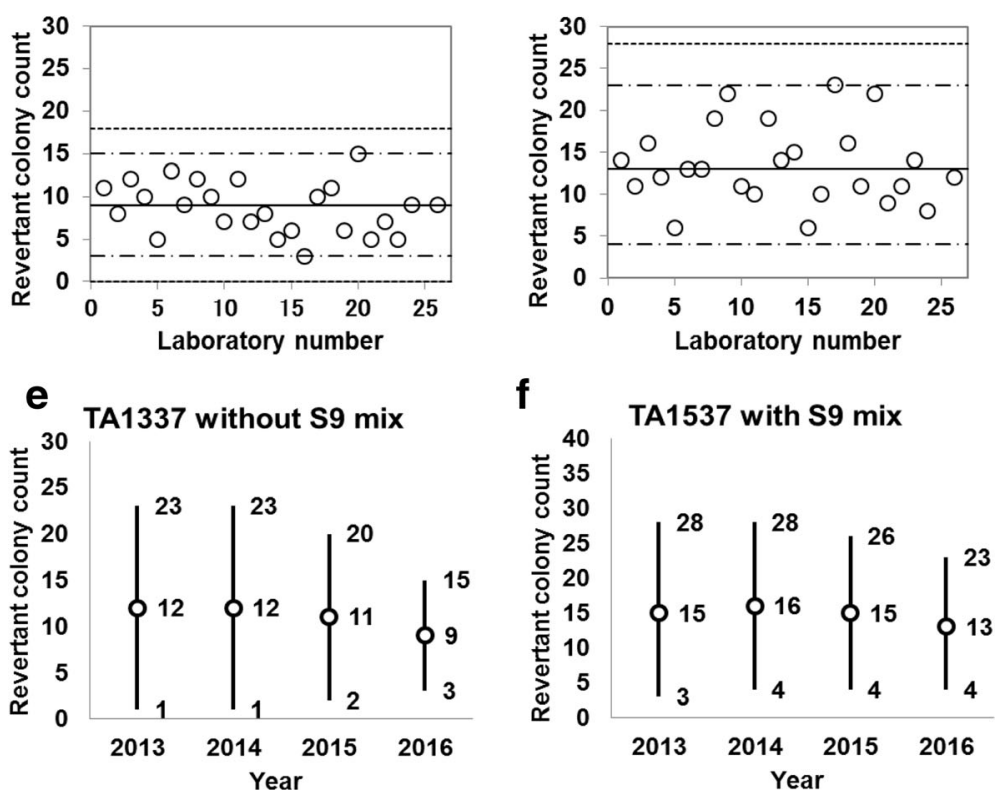

Fig. 4 Negative control counts, and their subsequent analysis, for Salmonella Typhimurium strain TA1537 with and without S9 mix. Scatter plots showing the negative control counts generated by each participating laboratory without (c) and with (d) S9 mix are also shown, where the inner horizontal lines (-.-) indicate the mean $\pm 2 \times$ standard deviation (SD), and outer horizontal lines (--) indicate the mean $\pm 3 \times$ SD. The data shown in panels (a) to (d) are taken from the study conducted in 2016. The mean $\pm 2 \times$ SD values for the negative control counts for each individual year without (e) and with (f) S9 mix are also presented

\section{Assay conditions}

The reverse mutation test was conducted using a preincubation procedure according to the members' own assay conditions, which had been established or confirmed to be valid through JEMS/BMS validation studies or seminars.

To obtain bacterial cells in early stationary phase, frozen stock cultures of each strain were inoculated into a conical flask or L-tube containing nutrient broth medium $(2.5 \%(w / v)$; Oxoid nutrient broth No. 2, Oxoid Ltd., Hampshire, United Kingdom), and then either stored for $>3 \mathrm{~h}$ at approximately $4{ }^{\circ} \mathrm{C}$ prior to culture, or cultured immediately in a shaking incubator for between 7 and $10 \mathrm{~h}$ at $37{ }^{\circ} \mathrm{C}$. Depending on the laboratory, the shaking incubators were set at various speeds (e.g. $140 \mathrm{rpm}$ for a $100-\mathrm{mL}$ conical flask, or 50 strokes/min for a 25-mL L-tube). The cell densities of each culture were confirmed to be $>1 \times 10^{9}$ cells $/ \mathrm{mL}$ by measuring optical density at $660 \mathrm{~nm}$. For the assays carried out with S9 mix, $0.1 \mathrm{~mL}$ of the negative (vehicle) or positive control solution was added to a test tube, to which $0.5 \mathrm{~mL}$ of $\mathrm{S} 9 \mathrm{mix}$ and $0.1 \mathrm{~mL}$ of bacterial culture were added. For assays carried out in the absence of S9 mix, $100 \mathrm{mM}$ sodium phosphate buffer ( $\mathrm{pH} 7.4$ ) was used in place of S9 mix. After mixing, the test tubes were preincubated for $20 \mathrm{~min}$ at $37{ }^{\circ} \mathrm{C}$ in a shaking water bath (between 70 and 150 strokes/min). Following preincubation, a $2-\mathrm{mL}$ volume of pre-warmed $\left(45^{\circ} \mathrm{C}\right)$ top agar was added to each tube and mixed. Each mixture was then immediately poured onto the surface of minimal-glucose agar plates. After the top agar hardened, plates were incubated for $48 \mathrm{~h}$ at $37{ }^{\circ} \mathrm{C}$. Each assay was conducted in duplicate (two plates per dose).

Water and DMSO were used as the negative (vehicle) controls for SA and for the other positive control 


\section{a}

WP2uvrA without S9 mix

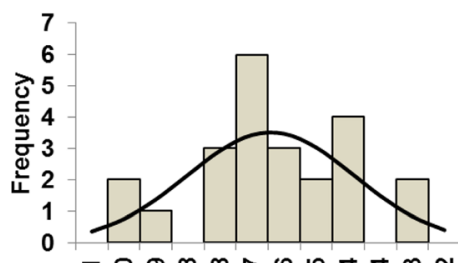

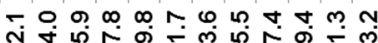

ํำ

Negative control count
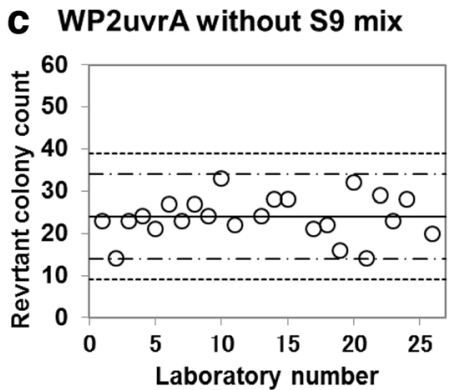

e WP2uvrA without $S 9$ mix

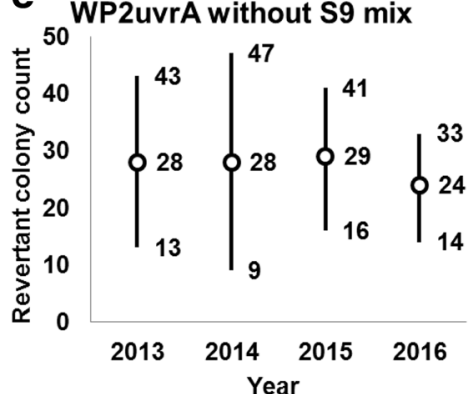

b

WP2uvrA with S9 mix

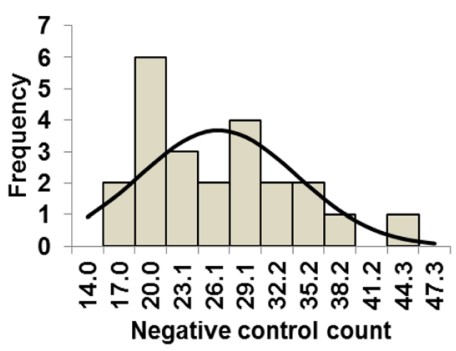

d WP2uvrA with S9 mix

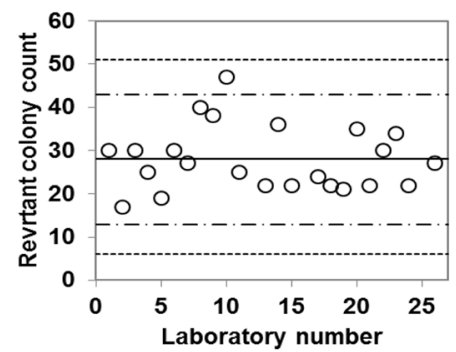

f

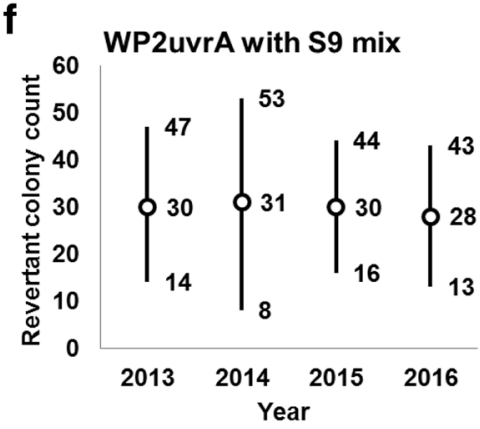

Fig. 5 Negative control counts, and their subsequent analysis, for Salmonella Typhimurium strain WP2uvrA with and without S9 mix. Scatter plots showing the negative control counts generated by each participating laboratory without (c) and with (d) S9 mix are also shown, where the inner horizontal lines (---) indicate the mean $\pm 2 \times$ standard deviation (SD), and outer horizontal lines (--) indicate the mean $\pm 3 \times$ SD. The data shown in panels (a) to (d) are taken from the study conducted in 2016. The mean $\pm 2 \times$ SD values for the negative control counts for each individual year without (e) and with (f) 59 mix are also presented

Table 1 Statistics for negative control data obtained in this study

\begin{tabular}{|c|c|c|c|c|c|c|c|c|c|c|}
\hline \multirow[t]{2}{*}{ Statistics } & \multicolumn{2}{|l|}{ TA100 } & \multicolumn{2}{|l|}{ TA98 } & \multicolumn{2}{|c|}{ TA1535 } & \multicolumn{2}{|c|}{ TA1537 } & \multicolumn{2}{|c|}{ WP2uvrA } \\
\hline & -59 & $+\$ 9$ & -59 & $+\$ 9$ & -59 & $+\$ 9$ & -59 & +\$9 & -59 & $+\$ 9$ \\
\hline No. of data & 26 & 26 & 26 & 26 & 25 & 25 & 25 & 25 & 23 & 23 \\
\hline Mean & 102 & 115 & 21 & 28 & 10 & 10 & 9 & 13 & 24 & 28 \\
\hline SD & 17.34 & 20.06 & 8.23 & 6.66 & 3.10 & 3.17 & 2.95 & 4.60 & 4.87 & 7.33 \\
\hline Variance & 312.57 & 418.66 & 70.36 & 46.06 & 10.01 & 10.48 & 9.08 & 22.01 & 24.75 & 56.23 \\
\hline Min & 69 & 78 & 12 & 17 & 6 & 5 & 3 & 6 & 14 & 17 \\
\hline Max & 132 & 156 & 40 & 42 & 19 & 18 & 15 & 23 & 33 & 47 \\
\hline Kurtosis & -0.63 & -0.64 & 0.58 & -0.73 & 0.88 & 0.64 & -0.63 & -0.24 & 0.04 & 0.29 \\
\hline Skewness & -0.09 & 0.30 & 1.22 & 0.40 & 0.98 & 0.80 & 0.15 & 0.54 & -0.25 & 0.82 \\
\hline 2SD- & 68 & 74 & 5 & 15 & 4 & 4 & 3 & 4 & 14 & 13 \\
\hline $2 \mathrm{SD}+$ & 137 & 156 & 37 & 42 & 17 & 17 & 15 & 23 & 33 & 43 \\
\hline 3SD- & 50 & 54 & -4 & 8 & 1 & 1 & 0 & 0 & 9 & 6 \\
\hline $3 \mathrm{SD}+$ & 154 & 175 & 46 & 48 & 20 & 20 & 17 & 27 & 38 & 50 \\
\hline
\end{tabular}

Min minimum count, max maximum count, $2 S D-$ mean $-2 \times$ standard deviation, $2 S D+$ mean $+2 \times$ standard deviation, $3 S D-$ mean $-3 \times$ standard deviation, $3 S D+$ mean $+3 \times$ standard deviation 
a TA100 without S9 mix, AF-2

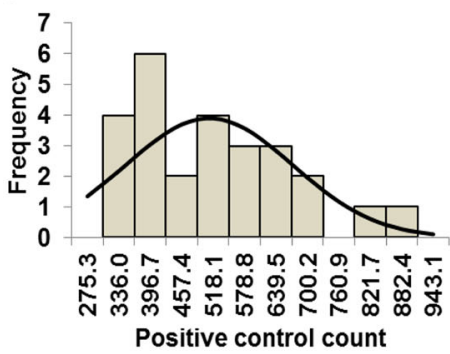

C

TA100 without 59 mix, AF-2

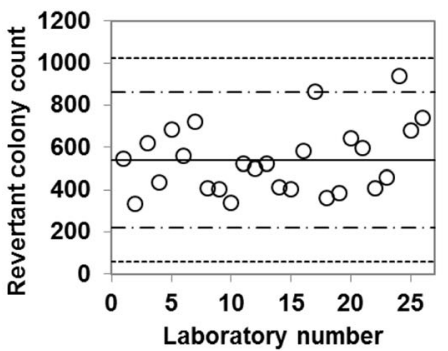

e TA100 without $S 9$ mix, AF-2

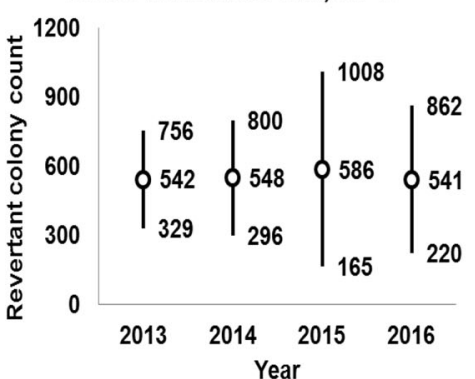

b TA100 with S9 mix, 2AA

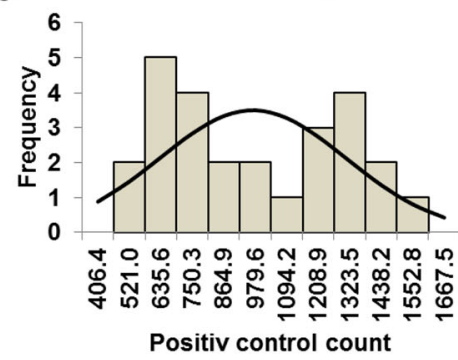

d

TA100 with S9 mix, 2AA

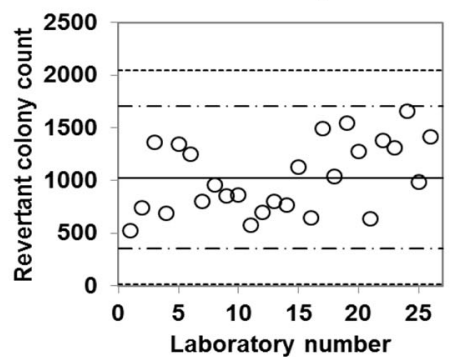

f

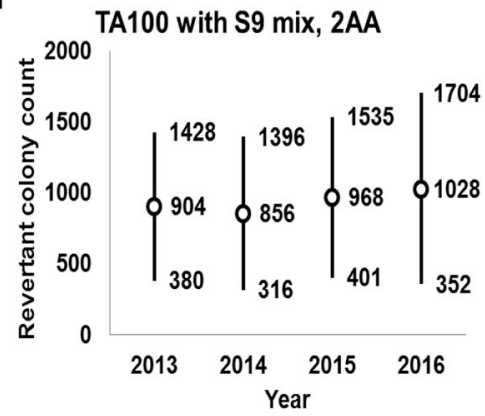

Fig. 6 Positive control counts, and their subsequent analysis, for Salmonella Typhimurium strain TA100 with and without S9 mix. Histograms show the negative control counts, and the curves indicate the expected values calculated based on the assumption that the negative control counts follow a normal distribution, without (a) and with (b) 59 mix. Scatter plots showing the negative control counts generated by each participating laboratory without (c) and with (d) S9 mix are also shown, where the inner horizontal lines (-.-) indicate the mean $\pm 2 \times$ standard deviation (SD), and outer horizontal lines (--) indicate the mean $\pm 3 \times$ SD. The data shown in panels (a) to (d) are taken from the study conducted in 2016. The mean $\pm 2 \times$ SD values for the negative control counts for each individual year without (e) and with (f) S9 mix are also presented. The doses used were $0.01 \mu \mathrm{g} /$ plate for AF-2 in the absence of $\mathrm{S} 9 \mathrm{mix}$, and $1.0 \mu \mathrm{g} / \mathrm{plate}$ for $2 \mathrm{AA}$ in the presence of $\mathrm{S} 9 \mathrm{mix}$

articles, respectively. The positive control articles and doses used for each strain are listed in Additional file 1: Table S1. The maximum dose for each positive control was as recommended by the Japan Industrial Safety and Health Association [9]. The number of revertant colonies induced at the highest doses were used as the positive control counts for the assay.

\section{Data analysis}

Mean and standard deviation (SD) were calculated from the experimental data generated by each laboratory using Excel (Microsoft, Redmond, WA, USA). The D'Agostino-Pearson and Kolmogorov-Smirnov tests were performed to evaluate the normality of data distribution, also using Excel [10]. A $p$-value $<0.05$ was considered to be statistically significant.

\section{Results and discussion \\ Negative (solvent) control data}

Histograms, along with their corresponding estimated frequency curves generated under the assumption that the counts were normally distributed, were generated from the negative control counts (the mean number of revertant colonies/plate) in the absence and presence of S9 mix for strains TA100 (Fig. 1a and b), TA98 (Fig. 2a and b), TA1535 (Fig. 3a and b), TA1537 (Fig. 4a and b), and WP2uvrA (Fig. 5a and b). These data were provided by 23-26 participating laboratories in 2016 . 


\section{a}

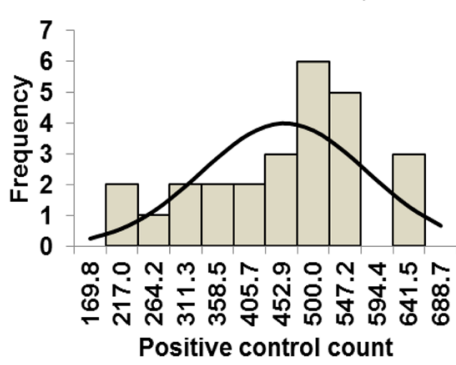

C TA98 without S9 mix , AF-2

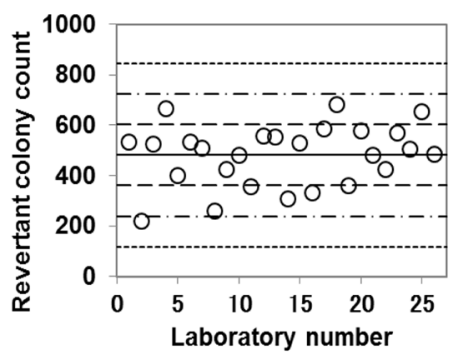

e TA98 without S9 mix, AF-2

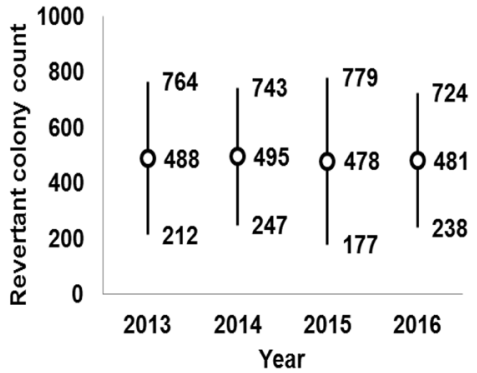

b TA98 with S9 mix, 2AA

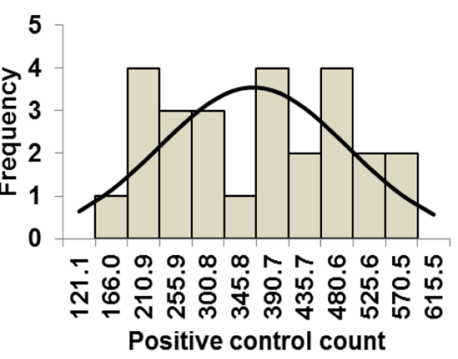

d TA98 with S9 mix, 2AA

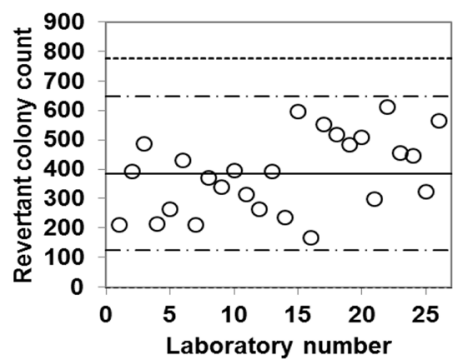

$\mathbf{f}$

Fig. 7 Positive control counts, and their subsequent analysis, for Salmonella Typhimurium strain TA98 with and without S9 mix. Histograms show the negative control counts, and the curves indicate the expected values calculated based on the assumption that the negative control counts follow a normal distribution, without (a) and with (b) S9 mix. Scatter plots showing the negative control counts generated by each participating laboratory without (c) and with (d) S9 mix are also shown, where the inner horizontal lines (---) indicate the mean $\pm 2 \times$ standard deviation (SD), and outer horizontal lines (--) indicate the mean $\pm 3 \times$ SD. The data shown in panels (a) to (d) are taken from the study conducted in 2016 . The doses used were $0.1 \mu \mathrm{g} /$ plate for AF-2 in the absence of S9 mix, and $0.5 \mu \mathrm{g} /$ plate for $2 \mathrm{AA}$ in the presence of S9 mix

D'Agostino-Pearson and Kolmogorov-Smirnov tests failed to reject the null hypothesis that the negative control counts were normally distributed for all five strains. In addition, the absolute values of the skewness and/or kurtosis for the five strains were less than 1.0, except for a value of 1.22 calculated for TA98 in the absence of S9 mix (Table 1). Most researchers tend to categorize absolute values of skewness and kurtosis of less than 1.0 as an indication of slight non-normality, values between 1.0 and about 2.3 as moderate non-normality, and values greater than 2.3 as severe non-normality. Therefore, the negative control counts of the strains with a relatively high number of negative control counts were deemed to be normally distributed (strains TA100, TA98, and WP2uvrA, both with and without S9 mix), as seen in
Fig. 1a and b, Fig. 2a and b, and Fig. 5a and b. In contrast, the negative control counts of the strains with a relatively low number of negative control counts can be deemed to approximately follow Poisson distribution, as the mean values were almost equal to the variance (strains TA1535 and TA1537, both with and without S9 mix), as seen in Table 1.

The negative control counts generated by each participating laboratory are shown in panels (c) and (d) of Figs. 1, 2, 3, 4, and 5. Almost all of the negative counts for each of the strains with and without S9 mix were within the range of the mean $\pm 2 \times \mathrm{SD}$, and counts from all laboratories were within the mean $\pm 3 \times \mathrm{SD}$, indicating that there were no outliers. As shown in panels (e) and (f) of Figs. 1, 2, 3, 4, and 5, there was little variance in 
a TA1535 without S9 mix, SA

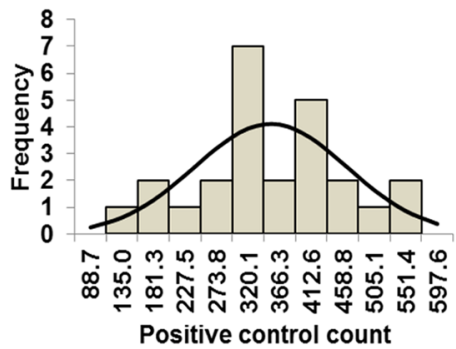

C TA1537 without S9 mix, 9AA

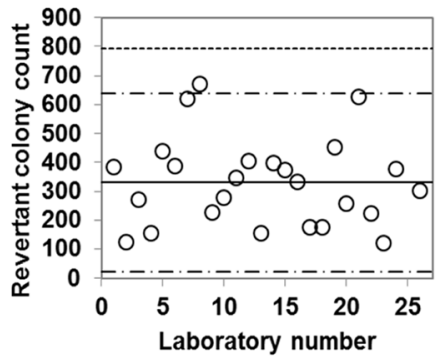

e TA1535 without S9 mix, SA

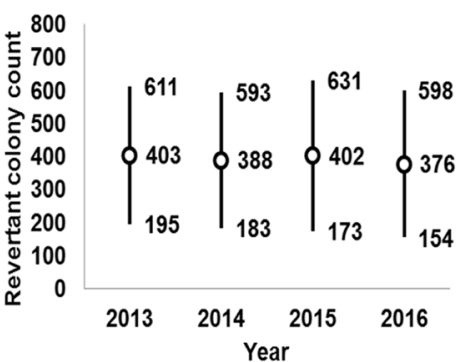

b TA1535 with S9 mix, 2AA

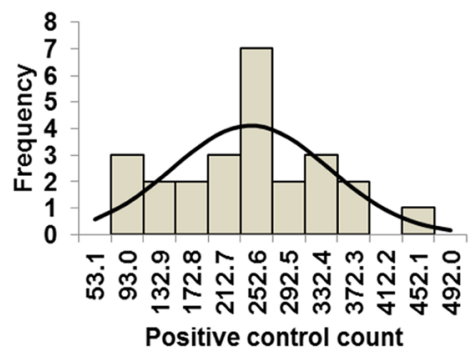

d TA1537 with S9 mix, 2AA

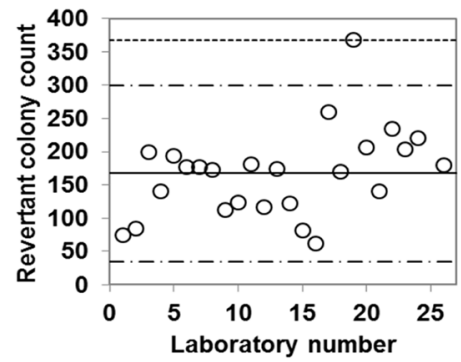

f

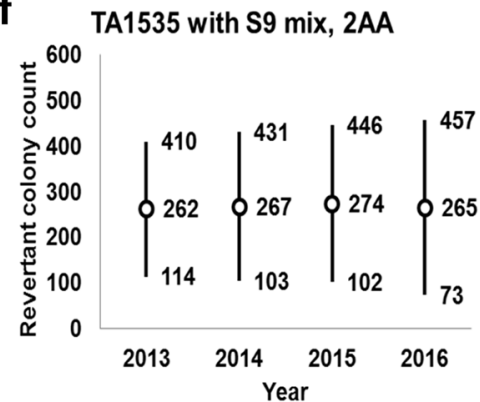

Fig. 8 Positive control counts, and their subsequent analysis, for Salmonella Typhimurium strain TA1535 with and without S9 mix. Histograms show the negative control counts, and the curves indicate the expected values calculated based on the assumption that the negative control counts follow a normal distribution, without (a) and with (b) 59 mix. Scatter plots showing the negative control counts generated by each participating laboratory without (c) and with (d) S9 mix are also shown, where the inner horizontal lines (---) indicate the mean $\pm 2 \times$ standard deviation (SD), and outer horizontal lines (--) indicate the mean $\pm 3 \times$ SD. The data shown in panels (a) to (d) are taken from the study conducted in 2016. The doses used were $0.5 \mu \mathrm{g} /$ plate for SA in the absence of $\$ 9 \mathrm{mix}$, and $2.0 \mu \mathrm{g} /$ plate for $2 \mathrm{AA}$ in the presence of S9 mix

the range of colony count values for each strain between each of the four years included in the study period. These findings indicate that laboratories using wellcontrolled assays carried out by proficient researchers can provide stable or consistent data. These negative control counts coincide with those reported previously $[4,5,9,11]$.

\section{Dose-response curves of positive control articles}

The dose-response curves for the five strains at three different doses of each control article (D1, D2, and D3; Additional file 1: Table S1) in the presence or absence of S9 mix are shown in Figs. 6, 7, 8, 9, and 10. The data for the dose-response curves were generated by 24-27 JEMS/BMS laboratories who participated in the validation study in 2016. All of the maximum doses designated "D3" (Additional file 1: Table S1) are those recommended for each positive control article by the Japan Industrial Safety and Health Association [9], and are in-line with doses frequently used in many Japanese laboratories. Linear relationships between the dose and the number of revertant colonies were observed for strains TA100, TA98, and TA1535, both with and without S9 mix (Additional file 2: Figure S1, Additional file 3: Figure S2, and Additional file 4: Figure S3), while exponential relationships between the dose and number of revertant colonies were observed for strains TA1537 and WP2uvrA, with and without S9 mix (Additional file 5: Figure S4 and Additional file 6: Figure S5).

Two laboratories showed a reduced number of revertant colonies at the maximum positive control article dose in the absence of S9 mix: strain TA98 treated with 
a TA1537 without S9 mix, 9AA

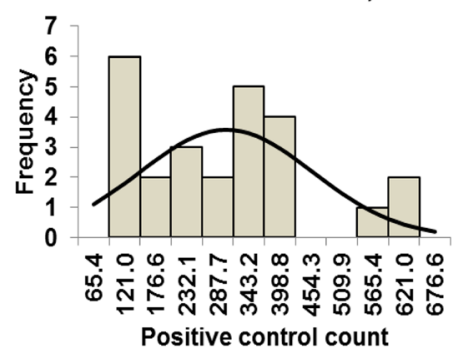

C

TA1537 without S9 mix, 9AA

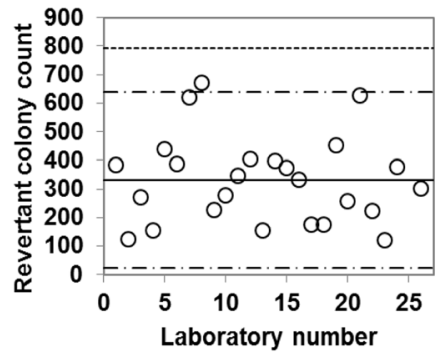

e TA1537 without S9 mix, 9AA

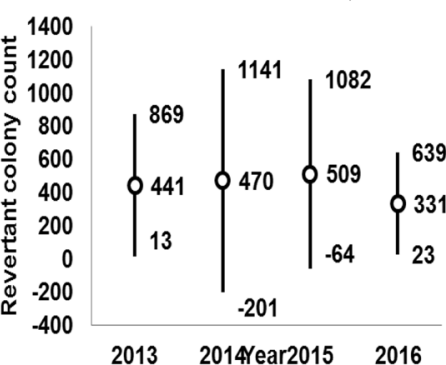

b TA1537 with S9 mix, 2AA

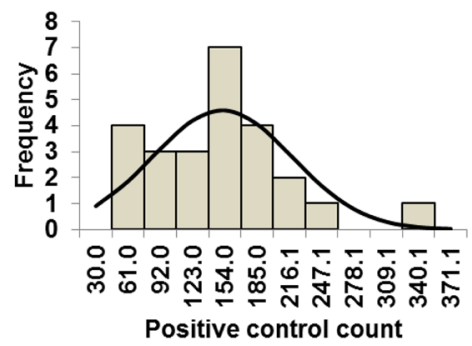

d

TA1537 with S9 mix, 2AA

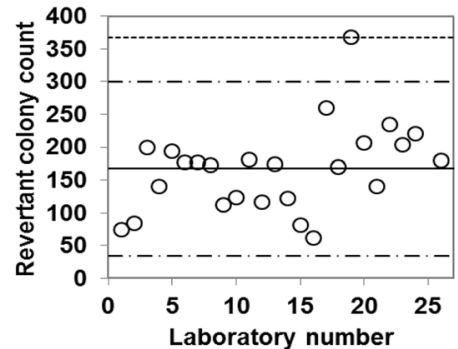

f

TA1537 with S9 mix, 2AA

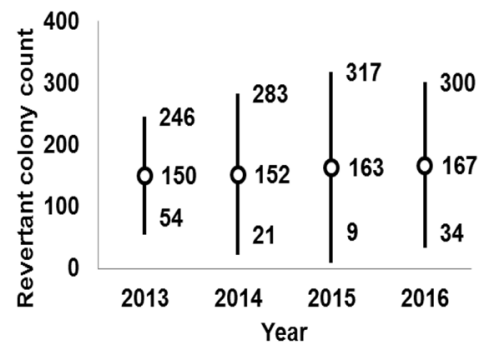

Fig. 9 Positive control counts, and their subsequent analysis, for Salmonella Typhimurium strain TA1537 with and without S9 mix. Histograms show the negative control counts, and the curves indicate the expected values calculated based on the assumption that the negative control counts follow a normal distribution, without (a) and with (b) S9 mix. Scatter plots showing the negative control counts generated by each participating laboratory without (c) and with (d) S9 mix are also shown, where the inner horizontal lines (---) indicate the mean $\pm 2 \times$ standard deviation (SD), and outer horizontal lines (--) indicate the mean $\pm 3 \times \mathrm{SD}$. The data shown in panels (a) to (d) are taken from the study conducted in 2016. The doses used were $80 \mu \mathrm{g} /$ plate for 9AA in the absence of 59 mix, and $2.0 \mu \mathrm{g} /$ plate for 2AA in the presence of 59 mix

AF-2 (Additional file 3: Figure S2), and strain TA1537 treated with 9AA (Additional file 5: Figure S4). The reduced activity seen at the highest dose of AF-2 is an example of why it may be best to use positive control doses that are not at or near the top of the doseresponse curves, but on the ascending portion of the curves. If the response falls within the flat portion at the top of the curve, then changes in the potency of the response may not be noticeable. Thus, this study provides corroborating evidence that the recommended positive doses outlined by the Japan Industrial Safety and Health Association [9] are appropriate. The mutagenic and cytotoxic potential of AF-2 is reported to vary depending on the pre-culture conditions. For example, this compound showed decreased mutagenicity but increased cytotoxicity towards bacteria cultured under anaerobic conditions compared with those cultured under aerobic conditions [11]. Therefore, the aberrant results obtained in the current study possibly indicate inappropriate preculture conditions. In the case of 9AA, we suspect some sort of technical error may account for the reduced number of revertant colonies obtained at the maximum dose by one laboratory.

\section{Positive control data}

Histograms and their corresponding estimated frequency curves (generated under the assumption that the counts were normally distributed) were produced from the positive control counts (the mean number of revertant colonies/plate) in the absence and presence of S9 mix for strains TA100 (Fig. 6a and b), TA98 (Fig. 7a and b), TA1535 (Fig. 8a and b), TA1537 (Fig. 9a and b), and 


\section{a}

WP2uvrA without S9 mix, AF-2

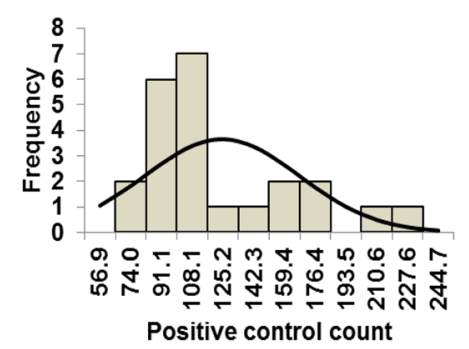

C WP2uvrA without S9 mix, AF-2

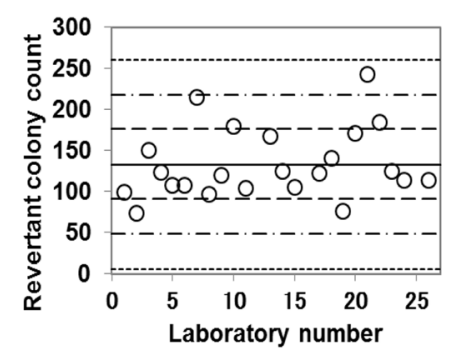

e WP2uvrA without $\mathrm{S9}$ mix, AF-2

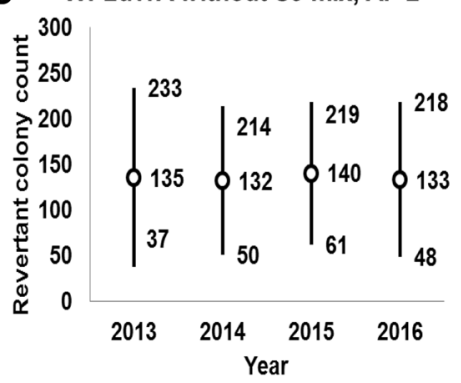

\section{b WP2uvrA with 59 mix, 2AA}

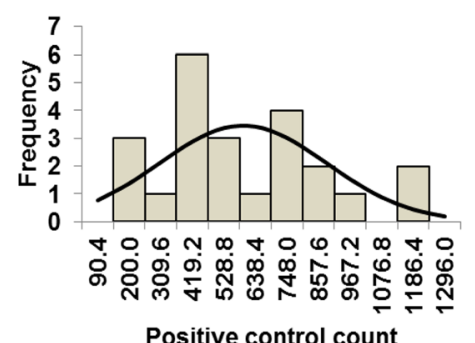

Positive control count

d WP2uvrA with 59 mix, 2AA

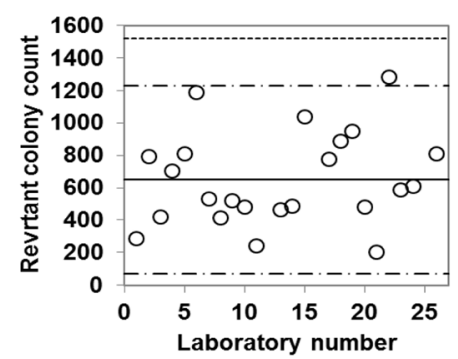

\section{f WP2uvrA with S9 mix, 2AA}

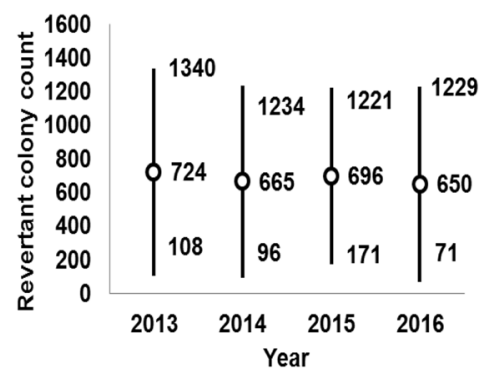

Fig. 10 Positive control counts, and their subsequent analysis, for Escherichia coli strain WP2uvrA with and without S9 mix. Histograms show the negative control counts, and the curves indicate the expected values calculated based on the assumption that the negative control counts follow a normal distribution, without (a) and with (b) 59 mix. Scatter plots showing the negative control counts generated by each participating laboratory without (c) and with (d) S9 mix are also shown, where the inner horizontal lines (---) indicate the mean $\pm 2 \times$ standard deviation (SD), and outer horizontal lines (--) indicate the mean $\pm 3 \times$ SD. The data shown in panels (a) to (d) are taken from the study conducted in 2016 . The doses used were $0.01 \mu \mathrm{g} /$ plate for SA in the absence of $\$ 9 \mathrm{mix}$, and $10 \mu \mathrm{g} /$ plate for 2AA in the presence of 59 mix

WP2uvrA (Fig. 10a and b). These data were provided by 24-27 participating laboratories in 2016.

D'Agostino-Pearson and Kolmogorov-Smirnov tests failed to reject the null hypothesis that the positive control counts were normally distributed for all five strains under all test conditions, except for strain TA1537 treated with 2AA in the presence of S9 mix. The absolute values of the skewness and kurtosis were less than 1.0 in most cases, although the observed values were slightly greater than 1.0 for TA100 and TA98 treated with $2 \mathrm{AA}$ in the presence of $\mathrm{S} 9 \mathrm{mix}$, and WP2uvrA treated with AF-2 in the absence of S9 mix (Table 2). Although the kurtosis value was 2.17 for TA1537 treated with $2 \mathrm{AA}$ in the presence of $\mathrm{S} 9 \mathrm{mix}$, it was less than the cut-off value of 2.3, values above which are indicative of severe non-normality (Table 2). None of the positive control counts showed Poisson distribution because the variances were much larger than the mean values. Therefore, we concluded that all positive control counts for all strains were approximately normally distributed.

The positive control counts generated by each participating laboratory are shown in panels (c) and (d) of Figs. $6,7,8,9$, and 10. Almost all of the positive control counts were within the range of the mean $\pm 2 \times \mathrm{SD}$, and all counts were within the mean $\pm 3 \times \mathrm{SD}$, indicating that there were no outliers. As shown in panels (e) and (f) of Figs. 6, 7, 8, 9 and 10, there was little variance in the range of colony count values for each strain between each of the four years included in the study period. As with the negative control counts, these findings indicate that laboratories with well-controlled assays and highly proficient staff can provide stable or consistent data. 
Table 2 Statistics for positive control data obtained in this study

\begin{tabular}{|c|c|c|c|c|c|c|c|c|c|c|}
\hline \multirow[t]{2}{*}{ Statistics } & \multicolumn{2}{|l|}{ TA100 } & \multicolumn{2}{|l|}{ TA98 } & \multicolumn{2}{|l|}{ TA1535 } & \multicolumn{2}{|l|}{ TA1537 } & \multicolumn{2}{|l|}{ WP2uvrA } \\
\hline & -59 & +59 & -59 & +59 & -59 & $+\$ 9$ & $-\$ 9$ & +59 & -59 & +59 \\
\hline $\begin{array}{l}\text { Positive control article and dose } \\
\text { ( } \mu \mathrm{g} / \text { plate) }\end{array}$ & AF-2 (0.01) & 2AA (1.0) & AF-2 (0.1) & 2AA (0.5) & SA (0.5) & 2AA (2.0) & 9AA (80) & 2AA (2.0) & AF-2 (0.01) & 2AA (10) \\
\hline No. of data & 27 & 27 & 27 & 27 & 26 & 26 & 26 & 26 & 24 & 24 \\
\hline Mean & 541 & 1028 & 481 & 386 & 376 & 265 & 331 & 167 & 133 & 650 \\
\hline SD & 157.23 & 331.4 & 119.21 & 128.07 & 108.98 & 93.99 & 150.7 & 65.38 & 41.60 & 282.98 \\
\hline Min & 336 & 521 & 217 & 166 & 135 & 93 & 121 & 61 & 74 & 200 \\
\hline Max & 937 & 1656 & 684 & 611 & 593 & 488 & 671 & 368 & 243 & 1285 \\
\hline Kurtosis & 0.13 & -1.28 & -0.30 & -1.09 & -0.03 & -0.01 & 0.00 & 2.17 & 0.80 & -0.23 \\
\hline Skewness & 0.80 & 0.25 & -0.45 & 0.05 & -0.21 & 0.15 & 0.67 & 0.89 & 1.05 & 0.55 \\
\hline 2SD- & 226 & 365 & 243 & 130 & 158 & 77 & 29 & 36 & 50 & 84 \\
\hline $2 S \mathrm{SD}+$ & 855 & 1691 & 719 & 643 & 594 & 453 & 632 & 298 & 216 & 1216 \\
\hline 3SD- & 69 & 34 & 123 & 2 & 49 & -17 & -122 & -29 & 8 & -199 \\
\hline $3 S D+$ & 1013 & 2022 & 839 & 771 & 703 & 547 & 783 & 363 & 258 & 1499 \\
\hline
\end{tabular}

Min minimum count, max maximum count, $2 S D-$ mean $-2 \times$ standard deviation, $2 S D+$ mean $+2 \times$ standard deviation, 3SD- mean $-3 \times$ standard deviation, $3 S D+$ mean $+3 \times$ standard deviation, AF-2 2-(2-furyl)-3-(5-nitro-2-furyl) acrylamide, 2AA 2-aminoanthracene, SA sodium azide, 9AA 9-aminoacridine hydrochloride

\section{Influence of different S9 manufactures/production lots on negative/positive control values and dose-response curves}

S9 fraction prepared from the livers of male SpragueDrawly rats pretreated with phenobarbital and 5,6benzoflavon was used by all participating laboratories in 2016. The S9 fractions were purchased from two manufacturers. Ten laboratories used S9 fraction manufactured by Kikkoman, while 16 laboratories used S9 fraction manufactured by Oriental Yeast. However, as shown in Additional file 7: Figure S6, no large differences were observed in the negative/positive control values or dose-response curves between the S9 manufactures or among production lots.

\section{Relationships between negative and positive control counts}

Scatter diagrams were generated to show the relationship between negative and positive control counts for each of the five strains with and without S9 mix (Additional file 8: Figure S7). The resulting low correlation coefficients suggested that there was no relationship between the negative and positive control counts.

\section{Conclusion}

The data presented here, collected from a series of validation studies conducted collaboratively by proficient JEMS/BMS members, will be of use in determining possible acceptance criteria to confirm or demonstrate laboratory proficiency in the reverse mutation test.

\section{Additional files}

Additional file 1: Table S1. Positive control articles and their doses used in this study (DOCX $18 \mathrm{~kb}$ )

Additional file 2: Figure S1. Dose-response curves of revertant Salmonella Typhimurium strain TA100 colonies following treatment with AF-2 in the absence of $\$ 9$ mix (a), or with 2AA in the presence of $\$ 9$ mix (b). Individual dose-response curves were generated using results produced by each participating laboratory in 2016 (different colors indicate different laboratories). The doses tested were $0.0025,0.005$, and $0.01 \mu \mathrm{g} /$ plate for AF-2, and 0.25, 0.5, and $1.0 \mu \mathrm{g} /$ plate for 2AA. (ODP $423 \mathrm{~kb}$ )

Additional file 3: Figure S2. Dose-response curves of revertant Salmonella Typhimurium strain TA98 colonies following treatment with AF-2 in the absence of 59 mix (a), or treatment with 2AA in the presence of 59 mix (b). Individual dose-response curves were generated using results produced by each participating laboratory in 2016 (different colors indicate different laboratories). The doses tested were $0.025,0.05$, and $0.1 \mu \mathrm{g} /$ plate for AF-2, and 0.125, 0.25, and $0.5 \mu \mathrm{g} /$ plate for 2AA. (ODP $434 \mathrm{~kb}$ )

Additional file 4: Figure S3. Dose-response curves of revertant Salmonella Typhimurium strain TA1535 colonies following treatment with SA in the absence of 59 mix (a), or with 2AA in the presence of 59 mix (b). Individual dose-response curves were generated using results produced by each participating laboratory in 2016 (different colors indicate different laboratories). The doses tested were $0.125,0.25$, and $0.5 \mu \mathrm{g} /$ plate for SA, and 0.5, 1.0, and $2.0 \mu \mathrm{g} /$ plate for 2AA. (ODP $411 \mathrm{~kb}$ )

Additional file 5: Figure S4. Dose-response curves of revertant Salmonella Typhimurium strain TA1537 colonies following treatment with 9AA in the absence of 59 mix (a), or with 2AA in the presence of $\$ 9$ mix (b). Individual dose-response curves were generated using results produced by each participating laboratory in 2016 (different colors indicate different laboratories). The doses tested were 20,40, and $80 \mu \mathrm{g}$ / plate for 9AA, and 0.5, 1.0, and 2.0 mg/plate for 2AA. (ODP 343 kb)

Additional file 6: Figure S5. Dose-response curves of revertant Escherichia coli strain WP2 UvrA colonies following treatment with AF-2 in the absence of S9 mix (a), or with 2AA in the presence of 59 mix (b). Individual dose-response curves were generated using results produced by each participating laboratory in 2016 (different colors indicate different laboratories). The doses tested were $0.0025,0.005$, and $0.01 \mu \mathrm{g} /$ plate for AF-2, and 2.5, 5.0, and $10 \mu \mathrm{g} /$ plate for $2 \mathrm{AA}$. (ODP $342 \mathrm{~kb})$ 
Additional file 7: Figure S6. Individual dose-response curves showing the positive control articles in the presence of $\mathrm{S} 9 \mathrm{mix}$ were generated from data obtained by each participating laboratory (each laboratory is indicated by a different color). The doses (in $\mu \mathrm{g} / \mathrm{plate}$ ) for each positive control article are the same as those shown in Figs. 6, 7, 8, 9, and 10. S9 fraction was purchased from Kikkoman or Oriental Yeast, and while some laboratories used that same lots, other laboratories used different lots from the same manufacturers. Laboratory identification numbers are indicated on the right-hand side of each figure. (ODP $516 \mathrm{~kb})$

Additional file 8: Figure S7. Comparison of the negative and positive control counts for each strain with or without $\mathbf{S 9}$ mix. The doses shown in Figs. $6,7,8,9$, and 10 were used for comparison. The $R^{2}$ values indicate the correlation coefficients of the linear regression lines. (ODP $62 \mathrm{~kb}$ )

\section{Abbreviations}

2AA: 2-aminoanthracene; 9AA: 9-aminoacridine hydrochloride; AF-2: 2-(2furyl)-3-(5-nitro-2-furyl) acrylamide; BMS: Bacterial Mutagenicity Study Group; DMSO: Dimethyl sulfoxide; GLP: Good Laboratory Practice; JEMS: Japanese Environmental Mutagen Society; OECD: Organisation for Economic Cooperation and Development; SA: Sodium azide; SD: Standard deviation

\section{Acknowledgments}

The authors are grateful to all JEMS/BMS members that participated in the validation studies. The authors also thank Dr. Dan Levy of Food and Drug Administration for his valuable comments, and Tamsin Sheen, PhD, from Edanz Group (www.edanzediting.com/ac) for editing a draft of this manuscript.

\section{Funding}

None.

\section{Availability of data and materials}

Not applicable.

\section{Authors' contributions}

MK is a chief organizer, and participated in study design and collection, analysis, and interpretation of the data. AH participated in study design and interpretation of data, and helped to draft and edit the manuscript. KS and TF drafted and edited the manuscript. TA, SH, KK, MN, MN, HS, and KW reviewed and critically discussed the manuscript. All authors read and approved the final manuscript.

Ethics approval and consent to participate

Not applicable.

\section{Consent for publication}

Not applicable.

\section{Competing interests}

The authors declare that they have no competing interests.

\section{Publisher's Note}

Springer Nature remains neutral with regard to jurisdictional claims in published maps and institutional affiliations.

\footnotetext{
Author details

${ }^{1}$ CMIC Bioresearch Center, CMIC Pharma Science Co., Ltd., Hokuto-shi, Yamanashi 408-0044, Japan. ${ }^{2}$ Division of Genetics and Mutagenesis, National Institute of Health Sciences, Setagaya-ku, Tokyo 158-8501, Japan. ${ }^{3}$ Scientfic Product Assessment Center, Japan Tobacco Inc., -shi, Yokohama, Kanagawa 227-8512, Japan. ${ }^{4}$ Otsuka Pharmaceutical Co., Ltd., Tokushima 771-0192, Japan. ${ }^{5}$ Global Quality Management Center, CANON INC., Kawasaki-shi, Kanagawa 213-8512, Japan. ${ }^{6}$ Hatano Research Institute, Food and Drug Safety Center, -shi, Hadano, Kanagawa 257-8523, Japan. ${ }^{7}$ Former University of Shizuoka, 52-1, Yada, Suruga-ku, Shizuoka-city, Shizuoka 422-8526, Japan. ${ }^{8}$ General Testing Research Institute, Japan Oilstuff Inspectors' Corporation, 2-15, 1-chome, Mikagetsukamachi, Higashinada-ku, Kobe-shi, Hyogo

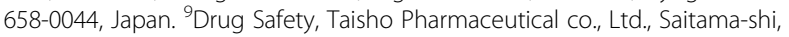

Saitama 331-9530, Japan. ${ }^{10}$ Tsukuba Drug Safety, Eisai Co., Ltd., Tsukuba-shi, Ibaraki 300-2635, Japan.

Received: 1 November 2017 Accepted: 7 February 2018

Published online: 04 April 2018

\section{References}

1. Honda H, Minegawa K, Fujita Y, Yamaguchi N, Oguma Y, Glatt H, Nishiyama N, Kasamatsu T. Modified Ames test using a strain expressing human sulfotransferase $1 \mathrm{C} 2$ to assess the mutagenicity of methyleugenol. Genes Environ. 2016:38:1

2. Fujii N, Yano S, Takeshita K. Selective enhancing effect of metal ions on mutagenicity. Genes Environ. 2016;38:21.

3. Hakura A, Hori Y, Uchida K, Sawada S, Suganuma A, Aoki T, Tsukidate K. Inhibitory effect of dimethyl sulfoxide on the mutagenicity of Promutagens in the Ames test. Genes Environ. 2010;32:53-60.

4. Maron DM, Ames BN. Revised methods for the Salmonella mutagenicity test. Mutat Res. 1983;113:173-215.

5. Mortelmans K, Zeiger E. The Ames Salmonella/microsome mutagenicity test. Mutat Res. 2000;455:29-60.

6. OECD. OECD Guidelines for the Testing of Chemicals / Section 4: Health Effects Test No. 471: Bacterial Reverse Mutation Test. OECD Publishing; 1997.

7. International Conference on Harmonisation (ICH) (2014). ICH M7 guideline, Assessment and Control of DNA Reactive (Mutagenic) Impurities in Pharmaceuticals to Limit Potential Carcinogenic Risk, Step 4 Available at: http://www.fda.gov/downloads/drugs/guidancecomplianceregulatory information/guidances/ucm347725.pdf. Accessed 25 Oct 2015.

8. Sugiyama K, Yamada M, Awogi T, Hakura A. The strain recommended for use in the bacteria reverse mutation test (OECD guideline 471) can be certified as non-genetically modified organisms. Genes Environ. 2016;38:1-3.

9. Ministry of Labor, Industrial Safety and Health Department. [Test Guidelines and GLP for Mutagenicity Test using Microorganisms in the Safety and Health Law]. Tokyo; Japan Industrial Safety and Health Association; 1991.

10. Zar JH. "Biostatistical Analysis," 4th ed. Upper Saddle River: Prentice-Hall; 1999.

11. Hakura A. Improved Ames test for genotoxicity assessment of drugs: preincubation assay using a low concentration of dimethyl sulfoxide. In: Yan Z, Caldwell GW, editors. Optimization in Drug Discovery: In Vitro Methods (second edition). Totowa: Humana Press; 2014. p. 325-36.

\section{Submit your next manuscript to BioMed Central and we will help you at every step:}

- We accept pre-submission inquiries

- Our selector tool helps you to find the most relevant journal

- We provide round the clock customer support

- Convenient online submission

- Thorough peer review

- Inclusion in PubMed and all major indexing services

- Maximum visibility for your research

Submit your manuscript at www.biomedcentral.com/submit
) Biomed Central 